\title{
THE IMPACT OF CROSSWALK DESIGN ON DRIVER PERFORMANCE: IMPLICATIONS FOR PEDESTRIAN SAFETY
}

\author{
Huizhong Guo ${ }^{1}$, Ning $\mathrm{Li}^{1}$, Linda Ng Boyle ${ }^{1}$, John K. Lenneman ${ }^{2}$, Tina Sayer ${ }^{2}$ \\ ${ }^{1}$ University of Washington, Seattle, WA, USA \\ ${ }^{2}$ Toyota Collaborative Safety Research Center, Ann Arbor, MI, USA \\ Email: linda@uw.edu
}

\begin{abstract}
Summary: Crosswalks are designed to enhance pedestrian visibility and right-ofway. This study examines driver performance at two different crosswalks with different pedestrian signal designs: (1) Rectangular Rapid Flashing Beacons (RRFBs) and (2) Pedestrian Hybrid Beacons (PHB). Mixed effects linear models showed that the drivers' speed significantly decreased when a signal was activated, with a larger decrease observed at a PHB signal when there was no lead vehicle. Mixed effects logistic models showed that drivers' likelihood to change distracting status was higher when a pedestrian signal was activated. The change could occur in both directions: from being engaged to not and from not engaged to distracted. Males and females responded differently to the RRFB in terms of speed differential and likelihood to engage in distractions from an undistracted state. In summary, PHB was able to decrease driver speed more effectively, while RRFB appeared to promote less travel delays for drivers.
\end{abstract}

\section{INTRODUCTION}

Pedestrian crashes have increased dramatically over the past few years. From 2009 to 2016, the total number of pedestrian fatalities in traffic crashes increased by $46 \%$, while motor fatalities increased by $10 \%$ for the same time period (NHTSA, 2018). Crosswalks designate an area for pedestrians to cross. However, pedestrians may incorrectly assume drivers will stop at these crosswalks (Mahalel \& Szternfeld, 1986). The likelihood of a vehicle-pedestrian crash is highly dependent on the frequency of observing a pedestrian (Sullivan \& Flannagan, 2002).

There are many types of crosswalk signals; two types used in Ann Arbor, MI are (1) Rectangular Rapid Flashing Beacons (RRFBs) and (2) Pedestrian Hybrid Beacons (PHBs). The RRFB is a user-actuated amber LED used to enhance pedestrian visibility at uncontrolled crosswalks (FHWA, 2009). RRFBs have been shown to increase vehicle yielding (slowing down) rates (Fitzpatrick, Brewer, \& Avelar, 2014). The PHB signal is design to alert drivers of pedestrians and guides them using a progression of four traffic phases: a flashing yellow for "slowing down", a solid yellow for "prepare to stop", a solid red for "stop", and a flashing red for "stop and proceed with caution". PHBs have also been shown to enhance drivers' yielding rate to pedestrians (Godavarthy \& Russell, 2016; Turner, Fitzpatrick, Brewer, \& Park, 2006). However, Godarvarthy and Russell also showed that some drivers do not fully understand the signal, possibly due to the complicated phase design of a PHB signal.

The goal of this paper is to examine changes in driver behavior as they approach a crosswalk installed with a RRFB or a PHB signal. The performance indicators will help explain the magnitude that a driver slows down and the likelihood that a driver engages in or disengages from a distracting or secondary task when the crosswalk signal is activated. The results of this 
study can provide insights for enhanced safety at these crosswalks.

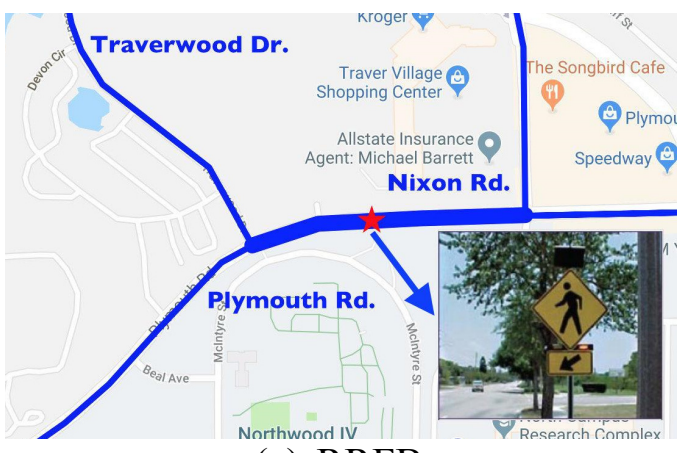

(a) RRFB

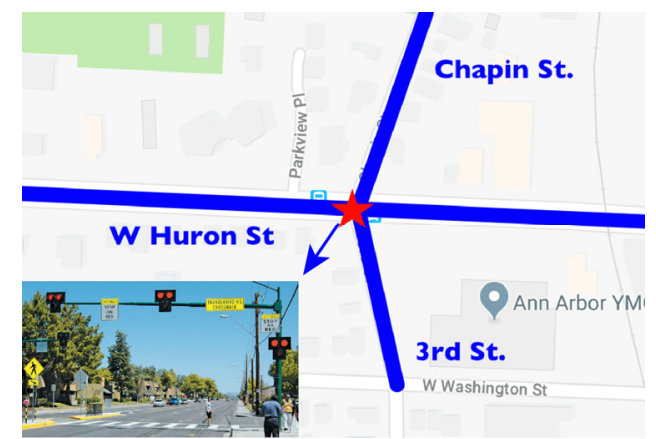

(b) PHB

Figure 1. Two examined crosswalks with RRFB and PHB (Google Maps), with overview of the two pedestrian signals on the map. Figures are from (FHWA, 2009) and (IIHS HLDI, 2010).

\section{METHODS}

\section{Data Source}

Naturalistic driving data from the Safety Pilot Model Deployment (SPMD) program was used for this study. The data were collected by the University of Michigan Transportation Research Institute (UMTRI) from August 2012 to June 2017. Participants had to be at least 18 years of age. No gender or maximum age restrictions were applied. SPMD sites included the northeast and a portion of the southeast corner of Ann Arbor, MI, but data were collected for any locations that participants traveled. The vehicles were equipped with Data Acquisition Systems (DASs) that also included data from videos recorded both inside and outside the vehicle.

\section{Scenarios}

For this study, a scenario is defined as an area that is traveled by a driver. We examined driver behavior at two scenarios.

RRFB at a midblock crosswalk. This study examined the eastbound direction between Traverwood Drive and Nixon Road (see Figure 1a), which included 400 meters of road. The crosswalk with the RRFB is located about 175 meters east of Traverwood Drive. This is a fivelane urban road with a shared left turn lane. The posted speed limit is 35 miles per hour (mph) or 15.64 meter per second $(\mathrm{m} / \mathrm{s})$.

PHB at an intersection. The PHB signal is located at the intersection of Huron Street and Chapin Street/3rd Street (see Figure 1b). We examined the eastbound direction of Huron Street approaching the intersection. Huron Street is a four-lane urban road with a posted speed limit of $35 \mathrm{mph}$ or equivalently $15.64 \mathrm{~m} / \mathrm{s}$. When the PHB is not activated, this intersection is an unsignalized intersection with stop signs on the two minor roads (i.e., Chapin Street and 3rd Street). 


\section{Data Analysis}

Dependent variables. We examined the change in driver behavior in response to a specific signal design using a "pre-scenario" and an "on-scenario" area. A "pre-scenario" refers to the area from 200 meters to 100 meters before the stop line. An "on-scenario" refers to the area from 100 meters before to 50 meters after the stop line. The 100 meters prior to the stop line is chosen based on the stopping sight distance (AASHTO, 2011). Using these segmentations, the outcomes of interest were:

- Speed difference score (continuous, $\mathrm{m} / \mathrm{s}$ ): calculated as the difference between the maximum speed of pre-scenario and the minimum speed of on-scenario. A positive speed difference score indicates a decrease in speed from pre- to on-scenario (drivers slow down), while a negative speed difference score means an increase (drivers speed up).

- Distraction engagement (yes/no): defined as whether a driver was engaging in any secondary (non-driving-related) tasks during on-scenario.

Explanatory variables. The following explanatory variables were considered:

- Signal activation (3 levels): "inactivated", "activated, with leading vehicle" and "activated, without leading vehicle".

- Traffic: (2 levels): used to identify whether the subject vehicle was influenced by surrounding traffic before the driver approached the crosswalk (yes, no). Influences can include a leading vehicle (in front of the subject or test vehicle) that slows down or merging into the subject's lane, resulting in the subject vehicle decelerating or steering.

- Gender (2 levels): female, male.

- Age group (4 levels): 21 to 30, 31 to 40, 41 to 50, 51 to 65 .

- Scenario (2 levels): RRFB, PHB

- Change in distraction (4 levels):

- Always: distracted in pre-scenario and on-scenario

- Disengaging: distracted in pre-scenario but undistracted in on-scenario

- Engaging: undistracted in pre-scenario but distracted in on-scenario

$\circ$ Never: undistracted in pre-scenario and on-scenario

Model. Mixed effects linear models were used to examine the magnitude of speed difference score given a pedestrian signal (activated or not) and the existence of leading vehicles (yes or no). Two mixed effects logit models were also used to examine driver engagement in distracted driving at these crosswalks. The first model shows the likelihood that a driver will engage in distracted driving during the on-scenario when they were initially undistracted in the prescenario. The second model shows the likelihood that a driver will continue engagement in distracted driving in the on-scenario when they were already distracted in the pre-scenario. For all models, random intercepts were included to account for individual differences. The best model was chosen by applying a backward elimination approach based on the log likelihood and Akaike Information Criterion (AIC). Data analyses were conducted in R (version 3.3.2) using the lme4 package (version 1.1-12). 


\section{RESULTS}

\section{Summary Statistics}

The SPMD data included 59 drivers that traveled through the RRFB and the PHB scenarios. Three drivers associated with the University of Michigan Transportation Research Institute (UMTRI) were removed. Twenty-eight drivers were excluded due to insufficient data (i.e., less than 3 associated events in any of the studied scenarios). The final dataset included 28 drivers with 400 events in the RRFB scenario and 430 events in the PHB scenario. Of these 28 drivers, age information was missing for two. The other 26 drivers were separated into four age groups: 21 to 30 years old ( 6 female and 3 male), 31 to 40 ( $2 \mathrm{~F}$ and $4 \mathrm{M}), 41$ to 50 ( $3 \mathrm{~F}$ and $3 \mathrm{M}$ ), and 51 to $60(4 \mathrm{~F}$ and $1 \mathrm{M})$.
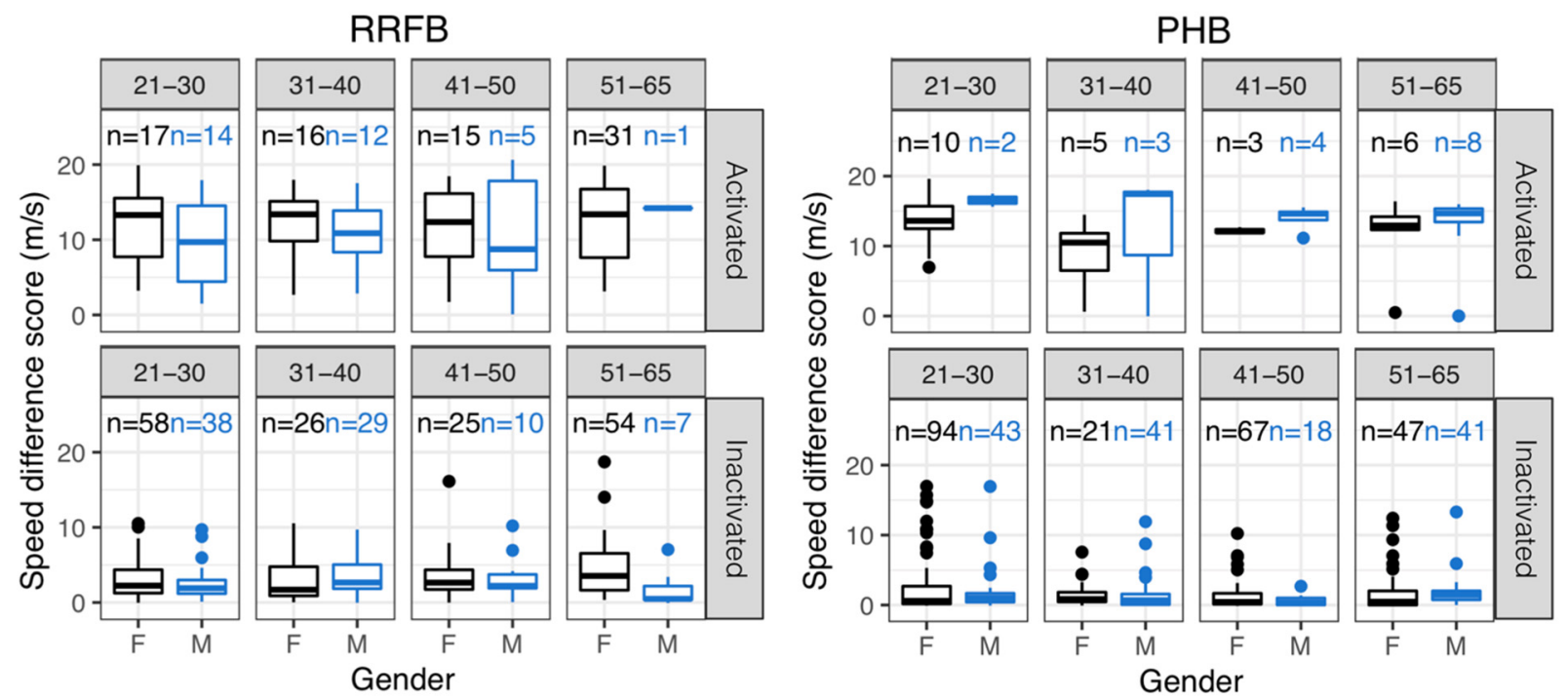

Figure 2. Speed difference score by gender and age group for RRFB and PHB (59 events with no age group or signal information were excluded from the figure)

The speed difference score ranges from $-0.07 \mathrm{~m} / \mathrm{s}$ to $22.04 \mathrm{~m} / \mathrm{s}$ in the RRFB scenario, and from $0.14 \mathrm{~m} / \mathrm{s}$ to $19.60 \mathrm{~m} / \mathrm{s}$ in the PHB scenario. When approaching the crosswalk, drivers generally slowed down or maintained their speed. For both the RRFB and PHB, the speed difference score was larger when the signal was activated (see Figure 2). No significant difference in speed difference score was observed across age groups. Some drivers had large speed difference scores in PHB even when the signal was not activated, most likely due to vehicles from the minor roads driving onto or crossing the major road. Females appeared to have a larger speed difference score than males for the RRFB scenario. For PHB, males had larger speed difference score when the signal was activated.

In RRFB, drivers were distracted both pre- and on-scenario in 118 events (29\%). They either disengaged or engaged in distraction from pre-scenario to on-scenario in 38 events $(10 \%)$ and 43 events (11\%), respectively. They were not distracted in 201 events (50\%). The three most frequently observed distracted activities in RRFB are interacting with passengers or pets (15\% in pre-scenario and $14 \%$ in on-scenario), talking/listening/singing ( $8 \%$ and $7 \%$ ), and personal hygiene (5\% and 6\%). In PHB, drivers were distracted both pre- and on-scenario in 116 events 
(27\%). They either disengaged or engaged in distraction from pre-scenario to on-scenario in 36 events $(9 \%)$ and 45 events (11\%), respectively. They were not distracted in 226 events $(53 \%)$. The three most frequently observed distracted activities in PHB are the same as those in RRFB, which are interacting with passengers or pets (14\% in pre-scenario and $15 \%$ in on-scenario), talking/listening/singing ( $6 \%$ and $6 \%$ ), and personal hygiene ( $4 \%$ and $5 \%$ ). There were 7 events with no distraction information in the PHB scenario.

\section{Model Results}

Table 1: Summary of three mixed effects models for predicting (1) speed difference score, likelihood of being distracted during on-scenario when (2) undistracted, or (3) distracted during pre-scenario.

\begin{tabular}{|c|c|c|c|c|c|c|}
\hline & \multicolumn{2}{|c|}{$\begin{array}{c}(1) \\
\text { Speed } \\
\text { difference } \\
\text { score }\end{array}$} & \multicolumn{2}{|c|}{$\begin{array}{c}(2) \\
\text { Undistracted } \\
\text { during } \\
\text { pre-scenario }\end{array}$} & \multicolumn{2}{|c|}{$\begin{array}{c}(3) \\
\text { Distracted } \\
\text { during } \\
\text { pre-scenario }\end{array}$} \\
\hline & Beta & P-value & Beta & P-value & Beta & P-value \\
\hline (Intercept) & 0.96 & 0.003 & -1.74 & $<0.001$ & 1.28 & $<0.001$ \\
\hline \multicolumn{7}{|l|}{ Age group (base: 21 to 30 ) } \\
\hline 31 to 40 & -0.04 & 0.909 & 0.61 & 0.363 & 0.12 & 0.769 \\
\hline 41 to 50 & -0.04 & 0.896 & 0.05 & 0.941 & 0.07 & 0.868 \\
\hline 51 to 65 & 0.32 & 0.296 & -0.92 & 0.217 & -0.32 & 0.358 \\
\hline Male & 0.32 & 0.331 & -0.05 & 0.930 & -0.48 & 0.115 \\
\hline \multicolumn{7}{|l|}{ Change in distraction (base: never) } \\
\hline Always & -0.17 & 0.642 & - & - & - & - \\
\hline Disengaging & -0.71 & 0.204 & - & - & - & - \\
\hline Engaging & -0.13 & 0.796 & - & - & - & - \\
\hline \multicolumn{7}{|l|}{ Signal activation (base: no) } \\
\hline Activated, with leading vehicle & 10.16 & $<0.001$ & 0.79 & 0.031 & -0.63 & 0.138 \\
\hline Activated, without leading vehicle & 9.71 & $<0.001$ & 0.16 & 0.778 & 0.24 & 0.718 \\
\hline Traffic influence - yes & 4.27 & $<0.001$ & 0.55 & 0.087 & 0.55 & 0.222 \\
\hline Scenario - RRFB & 1.72 & $<\mathbf{0 . 0 0 1}$ & -0.81 & 0.142 & 0.21 & 0.483 \\
\hline \multicolumn{7}{|l|}{ Interactions with RRFB } \\
\hline Age group 31 to 40 & - & - & -1.47 & 0.078 & - & - \\
\hline Age group 41 to 50 & - & - & -0.50 & 0.592 & - & - \\
\hline Age group 51 to 65 & - & - & 1.60 & 0.058 & - & - \\
\hline Male & -1.18 & 0.015 & 1.33 & 0.041 & - & - \\
\hline Change in distraction - always & 0.02 & 0.978 & - & - & - & - \\
\hline Change in distraction - disengaging & 0.67 & 0.410 & - & - & - & - \\
\hline Change in distraction - engaging & 1.50 & 0.051 & - & - & - & - \\
\hline Signal activation $-\mathrm{w} /$ leading vehicle & -0.53 & 0.515 & - & - & - & - \\
\hline Signal activation - w/o leading vehicle & -2.46 & 0.009 & - & - & - & - \\
\hline Traffic influence - yes & -1.28 & $\mathbf{0 . 0 3 8}$ & - & - & - & - \\
\hline Log likelihood & \multicolumn{2}{|c|}{-1951.69} & \multicolumn{2}{|c|}{-201.60} & \multicolumn{2}{|c|}{-153.02} \\
\hline $\mathrm{AIC}$ & \multicolumn{2}{|c|}{3945.39} & \multicolumn{2}{|c|}{431.20} & \multicolumn{2}{|c|}{326.05} \\
\hline
\end{tabular}

For both crosswalk scenarios (PHB and RRFB), there was a significantly larger speed difference score when a signal was activated or when traffic was present (see Table 1). When the signal was not activated, the speed difference score in the RRFB scenario was larger (by $1.72 \mathrm{~m} / \mathrm{s}$ ) when compared to the PHB scenario. However, when the signal was activated and no leading vehicle 
was present, the speed difference score was smaller in the RRFB than in the PHB (by 2.46$1.72=0.74 \mathrm{~m} / \mathrm{s}$ ). Compared to the PHB scenario, males and surrounding traffic in the RRFB decreased the speed difference score by $1.18 \mathrm{~m} / \mathrm{sec}$ and $1.28 \mathrm{~m} / \mathrm{sec}$, respectively.

For both PHB and RRFB, undistracted drivers during the pre-scenario had an increased odds of being distracted in the on-scenario when the pedestrian signal was on and when there was a leading vehicle (by a factor of $2.20=\exp (0.79))($ Table 1$)$. For males, the odds of being distracted in the on-scenario increased by a factor of $3.78(=\exp (1.33))$ in RRFB when compared to PHB. For drivers who were distracted pre-scenario, the mixed effects logit model did not reveal any factors that significantly impacted their distraction status on-scenario.

\section{DISCUSSION}

Drivers' behavior was examined on their approach to two different crosswalks: RRFB and PHB. Our study showed that drivers often slowed down when the signal was activated. When no leading vehicle was present, the speed difference score was larger in the PHB than in the RRFB. A possible reason is that the solid red and flashing red phases of a PHB signal required all approaching vehicles to stop. Vehicles that approach the RRFB can still pass through the crosswalk without slowing down even when the signal is flashing. The PHB signal appears to be a better pedestrian signal design in terms of forcing drivers to slow down before a crosswalk. However, the RRFB signal may be more efficient in that it induces less delay for drivers. On average, drivers took 24.7 secs to pass through a crosswalk when RRFB was activated. For an activated PHB crosswalk, the average time was 31.8 secs.

Drivers had larger speed difference scores in the PHB scenario (when compared to the RRFB) if traffic was present before the driver approached the crosswalk. The PHB examined was located at an intersection with no other traffic signals. Drivers on the major road may need to stop and wait for vehicles on minor road to pass through. This observation was observed in the model, which showed that drivers were impacted by traffic, but in different ways given the crosswalk type (RRFB or PHB).

Signal activation increased the odds of being distracted on-scenario for drivers who were undistracted pre-scenario. If drivers were distracted pre-scenario and there was a leading vehicle, signal activation decreased their odds of continuing to be distracted for the on-scenario. These findings show that drivers tend to have distraction migration when a signal is activated. It also indicates the two-side effect of a pedestrian signal control: it alerts distracted drivers and takes them back to undistracted driving status; on the other hand, it also triggers undistracted drivers to start doing some non-driving-related tasks when they slow down or stop.

In the PHB scenario, the drivers' gender did not significantly affect speed difference score or distraction behavior. In the RRFB scenario, males had relatively smaller speed difference scores. They were also more likely to become distracted as they travel from pre- to on-scenario in RRFB. These findings may be caused by the somewhat unbalanced distribution of driving events from different drivers in the dataset. One male driver, for example, has only 8 events in the RRFB scenario, but 54 events in the PHB scenario. Unbalanced distribution of repeated data is common in naturalistic driving studies due to the fact that repeated driving events are not 
controlled during the data collection process. Another implicit factor that could be related to the speed and behavior changes relate to drivers' personal driving styles.

There are many different crosswalk signal designs. This study examined only two types but showed differences in behavior between them. These differences could be associated with the signal design, the location of the crosswalk, or even the varying pedestrian behavior at each crosswalk. Some of the evaluation metrics used in this study could be used to examine other crosswalk signal designs. This would be very useful given that there are many crosswalk designs and it is not necessarily clear which ones are the most effective in reducing pedestrian crashes.

\section{ACKNOWLEDGEMENT}

This study was made possible through a contract with Toyota Collaborative Safety Research Center (CSRC). Any opinions, findings, and conclusions or recommendations expressed in this paper are those of the authors and do not necessarily reflect the views of Toyota.

\section{REFERENCES}

AASHTO. (2011). A Policy on Geometric Design of Highways and Streets, 2011. American Association of State Highway and Transportation Officials.

FHWA. (2009, May). Rectangular Rapid Flash Beacon (RRFB). Retrieved November 2018, from Federal Highway Administration: https://safety.fhwa.dot.gov/intersection/ conventional/unsignalized/tech_sum/fhwasa09009/

Fitzpatrick, K., Brewer, M., \& Avelar, R. (2014). Driver Yielding at Traffic Control Signals, Pedestrian Hybrid Beacons, and Rectangular Rapid-Flashing Beacons in Texas. Transportation Research Record: Journal of the Transportation Research Board, 2463(2463), 46-54. https://doi.org/10.3141/2463-06

Godavarthy, R. P., \& Russell, E. R. (2016). Study of Pedestrian Hybrid Beacon's Effectiveness for Motorists at Midblock Pedestrian Crossings. Journal of Traffic and Transportation Engineering (English Edition), 3(6), 531-539. https://doi.org/10.1016/j.jtte.2016.01.007

IIHS HLDI. (2010, September 28). Double-Red Signal Reduces Crashes at Crosswalks. Retrieved November 2018, from Insurance Institute for Highway Safety - Highway Loss Data Institute: https://www.iihs.org/iihs/sr/statusreport/article/45/10/4

Mahalel, D., \& Szternfeld, Z. (1986). Safety Improvements and Driver Perception. Accident Analysis \& Prevention, 18(1), 37-42. https://doi.org/10.1016/0001-4575(86)90034-5

NHTSA. (2018). Traffic Safety Facts - 2016 Data. Report number: DOT HS 812493. Washington, DC: National Highway Safety Administration.

Sullivan, J. M., \& Flannagan, M. J. (2002). The Role of Ambient Light Level in Fatal Crashes: Inferences from Daylight Saving Time Transitions. Accident Analysis and Prevention, 34(4), 487-498. https://doi.org/10.1016/S0001-4575(01)00046-X

Turner, S., Fitzpatrick, K., Brewer, M., \& Park, E. (2006). Motorist Yielding to Pedestrians at Unsignalized Intersections: Findings from a National Study on Improving Pedestrian Safety. Transportation Research Record: Journal of the Transportation Research Board, 1982, 112. https://doi.org/10.3141/1982-03. 